\title{
DETERMINAÇÃO NÃO DESTRUTIVA DO NITROGÊNIO TOTAL EM PLANTAS POR ESPECTROSCOPIA DE REFLECTÂNCIA DIFUSA NO INFRAVERMELHO PRÓXIMO
}

\author{
Kássio M. G. Lima, Marcello G. Trevisan, Ronei J. Poppi e João Carlos de Andrade* \\ Instituto de Química, Universidade Estadual de Campinas, CP 6154, 13084-971 Campinas - SP, Brasil
}

Recebido em 3/5/07; aceito em 17/8/07; publicado na web em 19/3/08

\begin{abstract}
NON-DESTRUCTIVE DETERMINATION OF TOTAL NITROGEN IN PLANTS BY DIFFUSE REFLECTANCE NEAR INFRARED SPECTROSCOPY. Diffuse reflectance near-infrared (DR-NIR) spectroscopy associated with partial least squares (PLS) multivariate calibration is proposed for a direct, non-destructive, determination of total nitrogen in wheat leaves. The procedure was developed for an Analytical Instrumental Analysis course, carried out at the Institute of Chemistry of the State University of Campinas. The DR-NIR results are in good agreement with those obtained by the Kjeldhal standard procedure, with a relative error of less than $\pm 3 \%$ and the method may be used for teaching purposes as well as for routine analysis.
\end{abstract}

Keywords: total nitrogen; near-infrared diffuse reflectance; teaching activity.

\section{INTRODUÇÃO}

A espectroscopia do infravermelho próximo (NIR), associada à calibração multivariada, é uma técnica bastante utilizada em diversas áreas. ${ }^{1-3}$ Por exemplo, os trabalhos de Willians e colaboradores $^{4}$ mostram que estes métodos são possibilidades seguras e rápidas na solução analítica de diversos problemas práticos, como os da determinação do teor de proteínas, umidade, carboidratos e gorduras em diferentes tipos de alimentos.

Dentre as técnicas de medida na região do infravermelho, destaca-se a espectroscopia de reflectância difusa no infravermelho próximo (DR-NIR), que já está bem fundamentada na literatura ${ }^{5}$ e que pode ser usada na determinação direta e não destrutiva de uma grande quantidade de substâncias em diferentes tipos de amostras, ${ }^{6-9}$ com economia de reagentes e de tempo. Ainda, as facilidades existentes atualmente para o acoplamento entre os equipamentos de medida e os microcomputadores, associadas à facilidade na estocagem de grandes quantidades de dados, possibilita maior rapidez nas medidas. Desta forma, as dificuldades anteriores na obtenção de espectros na região do infravermelho próximo foram contornadas. Estas características conferem vantagens particulares à técnica, tornando-a útil e muito interessante tanto em análises de rotina e como no controle on-line de processos industriais, com grande potencial para a aplicação dos conceitos da química verde.

Diferentes softwares (UNSCRAMBLER, NSAS, ISI, IDAS, Pirouette, etc.), que utilizam métodos matemáticos e estatísticos (métodos quimiométricos) ${ }^{10-13}$ para o máximo aproveitamento das informações obtidas, têm sido desenvolvidos e aplicados para tratar estes tipos de dados, sendo o da regressão por quadrados mínimos parciais (PLS) ${ }^{14}$ um dos mais empregados.

Embora nem sempre ocorram relações lineares entre os sinais espectroscópicos e as medidas da concentração total das espécies contidas na amostra, estes procedimentos já foram aplicados com sucesso em análises de matrizes complexas, fornecendo resultados rápidos, precisos e exatos. ${ }^{8,15,16}$

Por outro lado, apesar do crescente uso da espectroscopia de reflectância difusa no infravermelho próximo para a resolução de problemas práticos de rotina, relativamente pouca atenção tem sido

\footnotetext{
*e-mail: dandrade@iqm.unicamp.br
}

dada à esta técnica nos cursos de graduação das Instituições de Ensino Superior (IES) brasileiras. Assim, propõe-se um experimento simples, conduzido em um laboratório de ensino convencional, que aborda a teoria e prática da técnica DR-NIR, bem como a construção de um modelo multivariado para a determinação do teor de nitrogênio total em plantas (folhas de trigo), aplicando o modelo PLS no tratamento dos dados espectrais gerados a partir do conjunto de amostras. Os resultados espectroscópicos foram comparados com os obtidos por meio do método convencional de Kjeldhal, ${ }^{17,18}$ tomado como referência.

A estratégia utilizada compreende duas aulas teóricas (de $2 \mathrm{~h}$, cada) e uma aula prática $(4 \mathrm{~h})$. Na primeira aula teórica foram destacados os fundamentos e aspectos práticos da técnica NIR, e na segunda, a aquisição dos espectros NIR. O experimento requer a utilização de um espectrofotômetro que possa trabalhar na região do infravermelho próximo (entre 1000 e $2500 \mathrm{~nm}$ ), de um acessório de reflectância difusa e de um software quimiométrico. Os resultados apresentados neste trabalho foram obtidos pelos alunos da disciplina de Química Analítica Instrumental, oferecida pelo Instituto de Química da Universidade Estadual de Campinas, no primeiro semestre de 2005.

\section{PARTE EXPERIMENTAL}

\section{Amostras}

Para a construção dos modelos de regressão foram usadas 29 amostras de folhas de trigo, provenientes de diversos lugares, localizados em diferentes cidades do Estado de São Paulo, fornecidas pelo Instituto Agronômico de Campinas (IAC). As folhas foram lavadas com uma solução de água e detergente (1\% v/v) e enxaguadas várias vezes com água destilada e uma vez com água deionizada. Em seguida, foram colocadas em sacos de papel (para identificação), secas em estufa à $65{ }^{\circ} \mathrm{C}$ (mínimo de $24 \mathrm{~h}$ ), moídas em moinho tipo Wiley com abertura de $2 \mathrm{~mm}$ e peneiradas (177 $\mu \mathrm{m} ; 80 \mathrm{Mesh})$.

\section{Método de referência}

O método de Kjeldahl é empregado há muitas décadas na de- 
terminação de nitrogênio total em diferentes amostras. Pelo procedimento adotado no IAC para a determinação do nitrogênio total em plantas, ${ }^{18}$ faz-se inicialmente a digestão da amostra (folhas) com ácido sulfúrico, para transformar todo o nitrogênio em íons amônio. Esta conversão é feita com o emprego de catalisadores, para acelerar o processo de digestão.

Para a determinação da quantidade de amônio formado, o extrato (sulfúrico) da digestão é alcalinizado com hidróxido de sódio, produzindo amônia, que é destilada por arraste de vapor e recolhida em solução de ácido bórico $2 \% \mathrm{~m} / \mathrm{v}$,

$$
\mathrm{NH}_{3}+\mathrm{H}_{3} \mathrm{BO}_{3} \leftrightarrows \mathrm{H}_{2} \mathrm{BO}_{3}^{-}+\mathrm{NH}_{4}^{+}
$$

contendo uma mistura de indicadores (vermelho de metila e verde de bromocresol) para detecção do ponto final na retrotitulação dos íons borato formados, com solução padronizada de ácido sulfúrico.

$$
\mathrm{H}^{+}+\mathrm{H}_{2} \mathrm{BO}_{3}^{-} \leftrightarrows \mathrm{H}_{3} \mathrm{BO}_{3}
$$

A quantidade de ácido utilizada é proporcional ao teor de nitrogênio total, presente na amostra. Estas determinações foram efetuadas nos laboratórios do IAC e os resultados foram usados como referência.

\section{Espectroscopia no infravermelho próximo}

Os espectros foram obtidos em um espectrofotômetro UVVIS-NIR Varian, modelo Cary-5G, do Instituto de Química da UNICAMP, em triplicata na região espectral de 1000 a 2500 $\mathrm{nm}$, usando um acessório de reflectância difusa. Aparelhos mais simples também podem ser empregados. Foram feitas 16 varreduras espectrais para cada amostra sólida ( 80 Mesh), com resolução de $1 \mathrm{~nm}$, e os sinais foram expressos em $\log (1 / \mathrm{R})$. A importação e pré-tratamento dos dados, bem como a construção dos modelos quimiométricos foi feita pelo software Matlab (Mathworks Co.)

\section{Análise dos dados}

O método de regressão multivariada utilizado para o tratamento de dados deste estudo foi o método PLS (regressão por quadrados mínimos parciais). Este é um método bem conhecido ${ }^{19} \mathrm{e}$, como qualquer outro método de regressão, tem como objetivo encontrar uma relação entre a matriz $(\mathbf{X})$ contendo os espectros das amostras e o vetor que armazena as respectivas concentrações $(\mathbf{y})$. O resultado é uma equação semelhante à Equação 1:

$$
\mathbf{y}=\mathbf{X b}+\mathbf{e}
$$

onde e é o vetor que representa os erro do modelo e $\mathbf{b}$ é o vetor de regressão. O método PLS é especialmente indicado, quando $\mathbf{X}$ contém variáveis altamente correlacionadas (como os dados de espectroscopia descritos neste trabalho). Outra vantagem é que este método pode ser usado mesmo quando as amostras contêm interferentes (que estarão presentes no conjunto de calibração). No modelo PLS, a matriz $\mathbf{X}$ é decomposta em escores, $\mathbf{t}$, e pesos, $\mathbf{w}$, i.e. $\mathbf{X W}=\mathbf{T}$, onde $\mathbf{W}=(\mathrm{w} 1, \mathrm{w} 2, \ldots, \mathrm{w} k)$ é escolhido de maneira que $\mathbf{T}=$ $(\mathrm{t} 1, \mathrm{t} 2, \ldots, \mathrm{tk})$ apresente covariância máxima com $\mathbf{y}$ ( $k$ é o número de variáveis latentes). Assim, as informações espectrais e as concentrações são usadas ao mesmo tempo na fase de calibração. Um fator de suma importância na construção de um modelo PLS é a escolha do número de variáveis latentes $k(\mathrm{VL})$ a serem incluídas no modelo.

\section{Estatística}

O conjunto total de espectros foi dividido em dois subconjuntos: um de calibração e outro de validação externa. Para se determinar o número de Variáveis Latentes ${ }^{20}$ utilizadas no modelo, faz-se uma validação cruzada (validação interna) no conjunto de calibração: uma amostra do conjunto de calibração é excluída, o modelo é construído e então estimado o seu teor de nitrogênio total. O processo é repetido até que todas as amostras sejam previstas para 1 , $2, \ldots$ variáveis latentes. A habilidade do modelo de calibração para estimar (ou prever) o teor de nitrogênio total baseado nos dados dos espectros NIR gerados foi avaliada usando os erros de previsão e os coeficientes de correlação entre os valores dos teores de nitrogênio estimados pelo modelo, utilizando os espectros NIR e os valores do método de referência das amostras do conjunto de calibração. Os parâmetros de erro empregados foram: Soma dos quadrados dos erros de previsão (PRESS):

$$
\text { PRESS }=\sum_{i=1}^{n}\left(y_{i}-y_{i}\right)^{2}
$$

onde $n$ representa o número de amostras do conjunto de calibração, $\mathrm{y}_{i}$ é o valor de referência e $\mathrm{y}_{\mathrm{i}}^{\wedge}$ é o valor previsto pelo modelo para a iésima amostra.

Erro quadrático médio (MSE) ou a raiz quadrada do mesmo (RMSE) denominado também de erro padrão de previsão (SEP):

$$
M S E=\frac{\sum_{i=1}^{n}\left(y_{i}-y_{i}^{\wedge}\right)^{2}}{n-k-1}
$$

onde $\mathrm{k}$ é igual ao número de variáveis latentes.

$$
R M S E={\sqrt{\frac{\sum_{i=1}^{n}\left(y_{i}-y_{i}\right)}{n-k-1}}}^{2}
$$

Como estes erros podem ser medidos tanto para o conjunto de calibração quanto para o de validação externa, é comum adicionar no final da sigla destes erros a letra $\mathrm{C}$, indicando serem estes relativos à calibração C (MSEC ou RMSEC), ou P, quando forem relativos à previsão do conjunto de validação externa (MSEP ou RMSEP). Erro relativo percentual (ER (\%)) entre o método de referência e o método desenvolvido (NIR-PLS):

$$
\operatorname{ER}(\%)=\frac{\left(y_{i}-y_{i}\right)}{y_{i}} x 100
$$

\section{RESULTADOS E DISCUSSÃO}

Para a fase de calibração, os dados instrumentais obtidos a partir dos espectros NIR foram dispostos na forma de uma matriz $15 \mathrm{x}$ 1500 , que corresponde à matriz das variáveis independentes (matriz X). As colunas desta matriz são formadas pelos valores das intensidades em cada comprimento de onda do espectro (1000 a $2500 \mathrm{~nm}$; resolução de $4 \mathrm{~cm}^{-1}$ ) e o número de linhas é determinado pelo total de amostras selecionadas para esta fase. Uma outra matriz $15 \times 1$ é formada com os valores de nitrogênio total (\%), correspondentes às amostras da matriz $\mathbf{X}$, obtidos pelo método de Kjeldahl. Esta matriz 15 x 1 corresponde à matriz de variáveis dependentes (matriz $\mathbf{Y}$ ).

Na fase de previsão, os dados referentes aos 14 espectros NIR, 
não utilizados na fase de calibração, foram dispostos na forma de uma matriz 14 x 1500 correspondentes à matriz de variáveis independentes (matriz $\mathbf{X}_{\text {teste }}$ ). Novamente aqui tem-se que as colunas da matriz são formadas pelos valores de intensidades em cada comprimento de onda do espectro e o número de linhas corresponde ao número de amostras utilizadas para esta fase. As amostras escolhidas para a fase de previsão são aquelas que apresentam valores de nitrogênio total abrangendo toda a faixa de índices estudados e que são os mais distintos possíveis.

$\mathrm{Na}$ tentativa de minimizar os efeitos causados pela dificuldade de obtenção de um espectro ideal, ou seja, espectros sem variações aleatórias ou sistemáticas indesejáveis de diferentes origens, foram empregados tratamentos matemáticos nos espectros, antes da construção do modelo propriamente dito, para que estas variações não tivessem influência nos resultados finais. Foram utilizados os seguintes tratamentos em cada espectro: a transformação pela primeira derivada Savitzky Golay ${ }^{19}$ para minimizar os efeitos de espalhamentos de luz; o alisamento com splines ${ }^{21}$ e a média móvel para melhorar a razão sinal-ruído e, finalmente, a derivada primeira para a correção de deslocamento de linha de base. As absorbâncias referentes a cada comprimento de onda foram escaladas pela variância.

A escolha da região espectral foi feita de modo a se obter o melhor conjunto de comprimentos de onda que permitissem ao modelo de calibração minimizar os erros de previsão. As regiões espectrais usadas correspondem, principalmente, aos estiramentos simétricos e assimétricos dos grupos $\mathrm{N}-\mathrm{H}$, que estão compreendidas entre 1510 e $1979 \mathrm{~nm}$. Este critério foi avaliado pelos erros de previsão, como a raiz quadrada dos erros médios de previsão (RMSE).

Os espectros originais das 29 amostras analisadas, sem préprocessamento matemático, são mostrados na Figura 1. Para a correção dos efeitos de espalhamento multiplicativo e da flutuação da linha base, foi empregada a transformação pela primeira derivada Savitzky Golay ${ }^{19}$ (janela de 5 pontos) e o alisamento por "splines", ${ }^{21}$ aplicados nos espectros de reflectância originais. A Figura 2 mostra os espectros corrigidos, após este pré-processamento.

Os espectros corrigidos foram importados para o ambiente Matlab, a matriz de dados foi centrada na média e a avaliação dos modelos de calibração foi estudada utilizando-se o menor valor de PRESS obtido. O modelo que apresentou o menor PRESS de validação foi construído com cinco variáveis latentes, como indica o gráfico mostrado na Figura 3.

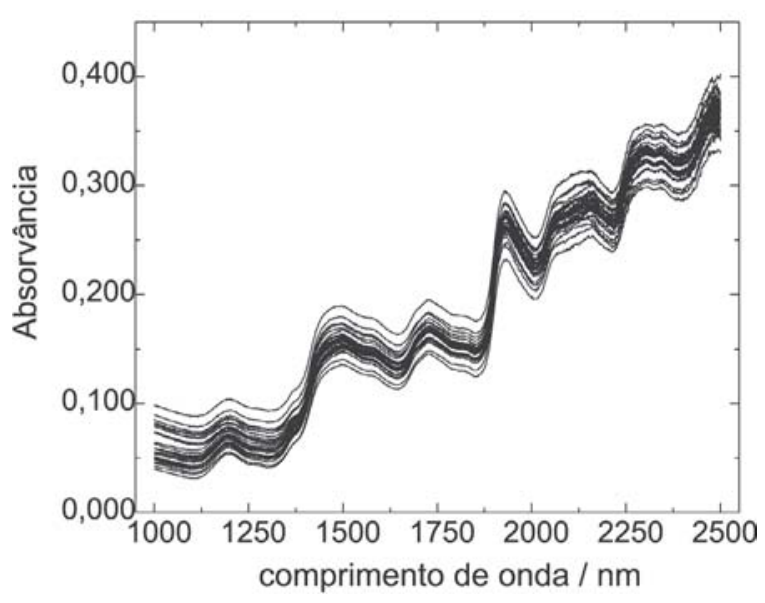

Figura 1. Conjunto dos espectros originais de reflectância difusa na região do NIR, sem transformações matemáticas

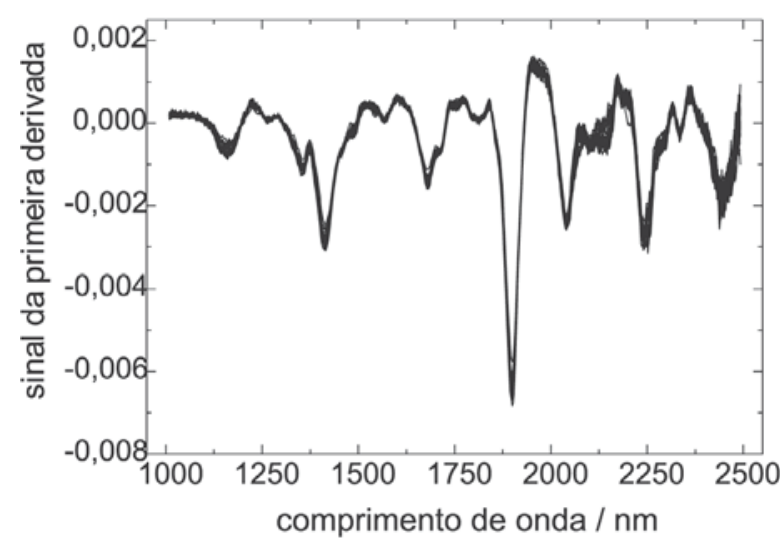

Figura 2. Conjunto dos espectros DR-NIR, após a correção pela $1^{a}$ derivada Savitzky Golay (janela de 5 pontos) e alisamento por spline

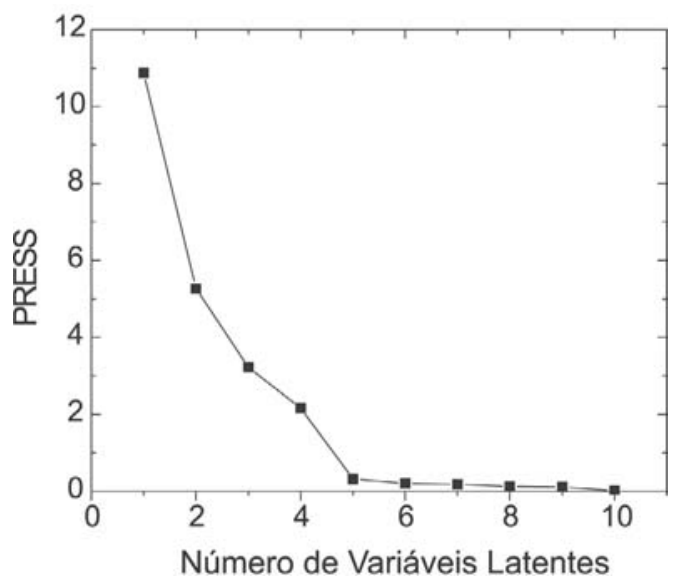

Figura 3. Gráfico de PRESS versus o número de variáveis latentes do modelo de calibração PLS

\section{Validação do modelo}

O modelo desenvolvido através das amostras de calibração foi validado por validação externa, utilizando-se medidas espectroscópicas DR-NIR de um conjunto de 14 amostras (do total de 29), em triplicata.

A Tabela 1 mostra, para fins comparativos, os valores experimentais, os valores médios previstos, os resíduos e o erro percentual para as amostras do conjunto de validação externa. Os erros relativos médios entre o método quimiométrico e o método de referência foram inferiores a $\pm 3,00 \%$, o que indica uma boa capacidade de previsão dos teores de nitrogênio total pelo método proposto para as amostras de folha de trigo.

A Figura 4 mostra a boa correlação existente entre os valores médios do teor de nitrogênio do conjunto de amostras e os valores experimentais verificados para as 14 amostras utilizadas no conjunto de validação externa. A baixa dispersão dos valores de previsão em torno da curva revela uma boa habilidade de previsão do modelo. Através do mesmo gráfico, é possível verificar a alta correlação entre os valores previstos pelo modelo proposto e seus valores de referência (exatidão), evidenciando a ausência de erros sistemáticos nos resultados.

A precisão das medidas (em triplicata) obtidas através do modelo PLS foi comparada com a observada para o método de refe- 
Tabela 1. Comparação dos resultados de nitrogênio total (g/1000 g) para as amostras do conjunto de validação externa obtidos pelos métodos de Kjeldhal e NIRS - PLS aplicando o modelo de regressão PLS

\begin{tabular}{|c|c|c|c|}
\hline $\begin{array}{l}\text { Valor médio } \\
\text { previsto } \\
\text { (Método DR- } \\
\text { NIR/PLS) }\end{array}$ & $\begin{array}{c}\text { Valor } \\
\text { experimental } \\
\text { (Método de } \\
\text { Kjeldhal) }\end{array}$ & Resíduo & $\begin{array}{c}\text { Erro } \\
(\%)\end{array}$ \\
\hline 49,40 & 49,50 & $-0,09$ & $-0,18$ \\
\hline 51,26 & 51,20 & 0,06 & 0,12 \\
\hline 49,20 & 49,10 & 0,10 & 0,21 \\
\hline 47,81 & 47,90 & $-0,08$ & $-0,17$ \\
\hline 33,71 & 33,90 & $-0,18$ & $-0,53$ \\
\hline 36,75 & 37,50 & $-0,74$ & $-1,98$ \\
\hline 35,01 & 34,10 & 0,91 & 2,67 \\
\hline 39,83 & 39,80 & 0,03 & 0,08 \\
\hline 45,82 & 46,00 & $-0,17$ & $-0,37$ \\
\hline 36,04 & 36,00 & 0,00 & 0,11 \\
\hline 37,51 & 38,30 & $-0,78$ & $-2,04$ \\
\hline 41,31 & 41,30 & 0,01 & 0,02 \\
\hline 37,54 & 36,50 & 1,04 & 2,87 \\
\hline 37,89 & 38,30 & $-0,41$ & $-1,06$ \\
\hline
\end{tabular}

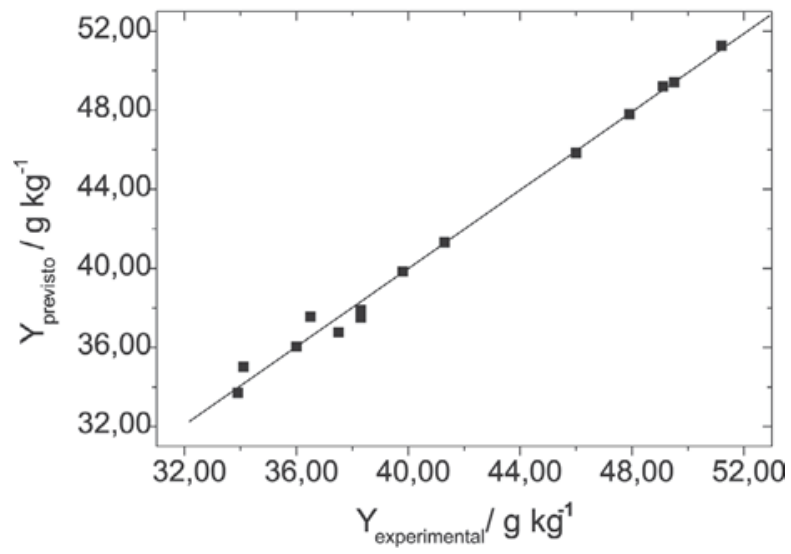

Figura 4. Correlação entre os valores os valores previstos $\left(Y_{\text {previsto }}\right)$ e os experimentais ( $\left.Y_{\text {experimental }}\right)$, para o conjunto de validação externa do modelo, com 5 variáveis latentes

rência (tomadas em duplicatas). Para comparar a precisão entre estes dois conjuntos de dados foi aplicado o teste-F, utilizando a distribuição $\mathrm{F}$ com o nível de 5\% de probabilidade. Excetuando uma medida, todas as outras mostram que não há diferença significativa entre a precisão dos dados obtidos pelo modelo e aquela obtida a partir do método de referência, para o nível de $5 \%$ de probabilidade.

\section{CONCLUSÃO}

O experimento proposto permite introduzir conceitos relacionados à espectroscopia no infravermelho próximo e aos métodos de regressão, através de uma aplicação analítica prática usando medidas de reflectância difusa. $\mathrm{O}$ procedimento proposto mostrouse adequado como prática experimental em uma disciplina de análise instrumental para alunos do curso de bacharelado em química e pode ser aplicado em análises de rotina. Este experimento pode ser executado em um período de $4 \mathrm{~h}$ de laboratório, incluindo a aquisição dos espectros, e vem de encontro à necessidade de se desenvolver material didático direcionado aos cursos de graduação, englobando um maior número de conceitos e utilizando técnicas instrumentais muito empregadas atualmente, tanto em pesquisa como nos laboratórios do setor produtivo.

\section{AGRADECIMENTOS}

À CAPES/CNPq e ao Programa de Estágio a Docência (PED) do Instituto de Química da UNICAMP, pela bolsa recebida pelo doutorando K. M. G. Lima.

\section{REFERÊNCIAS}

1. Wang, L.; Lee, F. S. C.; Wang, X. R.; Food Chem. 2006, 95, 529.

2. Rui, Y. K.; Luo, Y. B.; Huang, K. L.; Zhang, L. D.; Spectrosc. Spectral Anal. 2005, 25, 1581.

3. McClure, W. F.; J. Near Infrared Spectrosc. 2003, 11, 487.

4. Willians, P.; Norris, K.; Near Infrared Technology in the Agricultural and Food Industries, AACC Press: Minnesota, 1990.

5. Hooton, D. E.; Cereal Food World 1978, 23, 176.

6. Miyamae, Y.; Yamakawa, Y.; Ozaki, Y.; Appl. Spectrosc. 2007, 67, 212.

7. Jones, P. D.; Schimleck, L. R.; Peter, G. F.; Wood Sci. Technol. 2006, 40, 709 .

8. Masserschmidt, I.; Cuelbas, C. J.; Poppi, R. J.; De Andrade, J. C.; De Abreu, M. F.; Davanzo, C. U.; J. Chemom. 1999, 13, 265.

9. Mello, C.; Poppi, R. J.; De Andrade, J. C.; Canterella, H.; Analyst 1999, 124, 1669.

10. Geladi, P. Em Near Infrared Spectroscopy: The Future Waves; Davies, A. M.; Willians, P., eds.; NIR Publications: Chichester, 1996, cap. 29.

11. Uddin, M.; Okazaki, E.; Ahmad, M. U.; Fukuda, Y.; Tanaka, M.; Food Control 2006, 17, 660 .

12. Galvão, R. K. H.; Araújo, M. C. U.; Martins, M. D.; José, G. E.; Pontes, M. J. C.; Silva, E. C.; Saldanha, T. C. B.; Chemom. Intell. Lab. Syst. 2006, $81,60$.

13. Arana, I.; Jaren, C.; Arazuri, S.; J. Near Infrared Spectrosc. 2005, 13, 349.

14. Yu, H. Y.; Ying, Y. B.; Fu, X. P.; Lu, H. S.; J. Near Infrared Spectrosc. 2006, 14, 37.

15. Fidencio, P. H.; Poppi, R. J.; De Andrade, J. C.; Cantarella, H.; Commun. Soil Sci. Plant Anal. 2002, 33, 1607.

16. Fidencio, P. H.; Poppi, R. J.; De Andrade, J. C.; Anal. Chim. Acta 2002, 453, 125.

17. Wiles, P. G.; Gray, I. K.; Kissling, R. C.; J. AOAC Int. 1998, 81, 620.

18. IAC; Protocolo de Análise: Determinação de nitrogênio em material vegetal por destilação, SFSNP: Campinas, 1990.

19. Delwiche, S. R.; Reeves, J. B.; J. Near Infrared Spectrosc. 2004, 12, 177.

20. Turak, F.; Ozgur, M. U.; Ercag, A.; Bozdogan, A.; Rev. Anal. Chem. 2006, 25, 295.

21. Faber, N. M.; Appl. Spectrosc. 1999, 53, 1011. 